\title{
El defecto de Gerbode y traumatismo torácico cerrado. Presentación de un caso y revisión de la literatura
}

\author{
José G. Somocurcio ${ }^{1}$, Gamero Álvarez ${ }^{1}$, Alfredo Sotomayor ${ }^{1}$, \\ Robert Torrejón ${ }^{2}$, Rafael Vicuña ${ }^{3}$
}

Resumen

Palabras clave
Presentamos un caso raro de comunicación ventrículo izquierdo-aurícula derecha (defecto tipo Gerbode) asociado a traumatismo torácico cerrado, que incluye los hallazgos ecocardiográficos e intraoperatorios. El paciente fue operado exitosamente. Ocasionalmente, se encuentra las comunicaciones ventrículo izquierdo-aurícula derecha adquiridas en la población adulta que pueden suceder como resultado de endocarditis o en el reemplazo de la válvula aórtica.

Defectos del septum; traumatismos torácicos; cardiopatías congénitas.
Gerbode defect and blunt chest trauma. Presentation of a case and literature review

Abstract

We present a rare case of left ventricular-to-right atrial communication (Gerbode type defect) associated with blunt thoracic trauma, including echocardiogram and surgical findings. The patient had a successful intervention. Acquired left ventricular-to-right atrial shunts are encountered occasionally in the adult population as a result of aortic valve replacement endocarditis.

Key words: Heart septal defects; thoracic injuries; heart defects, congenital.

\section{INTRODUCCIÓN}

El defecto de Gerbode es la variación de un defecto septal ventricular membranoso, que deriva parte del flujo ventricular izquierdo dentro de la aurícula derecha. Esto es anatómicamente posible por la implantación más distal de la válvula tricúspide con respecto de la válvula mitral.

1 Departamento Académico de Cirugía, Facultad de Medicina Universidad Nacional Mayor de San Marcos. Lima, Perú.

2 Facultad de Medicina, Universidad Nacional Federico Villareal. Lima, Perú.

3 Médico residente de la Universidad Nacional Mayor de San Marcos, especialidad de Cirugía de Tórax y Cardiovascular. Lima, Perú.
El diagnóstico diferencial de esta entidad infrecuente se realiza con los defectos septales ventriculares membranosos y es pocas veces comunicado en la literatura.

El objetivo de esta comunicación es la presentación de un caso, diagnosticado y operado exitosamente en el Servicio de Cirugía de Tórax y Cardiovascular del Hospital Nacional Hipólito Unanue de Lima. Se hace la revisión de la literatura sobre el tema.

\section{CASO CLÍNICO}

Paciente de 22 años de edad de sexo masculino, natural y residente en Lima, con grado de instrucción secundaria, desempleado, que ingresa por el servicio de emergencia con síntomas cardíacos congestivos. El paciente estaba consciente y refirió que sus molestias se habían iniciado de manera brusca y de modo progresivo, al haber sufrido caída de aproximadamente $2,5 \mathrm{~m}$ de altura y ser (agredido) golpeado en la cara y otras partes el cuerpo con ladrillos y otros objetos contundentes. En el servicio de emergencia se le practicó suturas de heridas contusas de mentón y labios, permane- 
ciendo en observación por 48 horas, con el diagnóstico de 'policontuso y TEC leve-moderado'. La tomografía axial computada cerebral y la ecografía abdominal fueron informadas como normales. La radiografía estándar de tórax descartó la presencia de hemotórax y neumotórax. Cinco días después de haber sido dado de alta del servicio de emergencia, acudió a consulta externa neumológica, refiriendo dolor precordial, tos con expectoración mucosa, ocasionalmente hemoptoica, y disnea a los esfuerzos moderados. La radiografía del tórax mostró 'contusión pulmonar moderada y proceso inflamatorio parenquimal'. El examen para bacilo de Koch directo del esputo fue negativo y se auscultó un soplo sistólico mesocárdico y bradicardia.

Fue hospitalizado en nuestro servicio, siete meses después del inicio de estas molestias, refiriendo disnea a los pequeños esfuerzos. Registraba entre sus antecedentes haber sido tratado de otitis media supurada a la edad de 3 años. Tenía un hermano mayor fallecido por tuberculosis multidrogo-resistente; negaba así mismo tuberculosis pulmonar, hepatitis, entre otros, y no había tenido limitaciones físicas para los esfuerzos, siendo practicante habitual de deportes exigentes, como el fútbol. No había tenido hospitalizaciones previas ni operaciones.

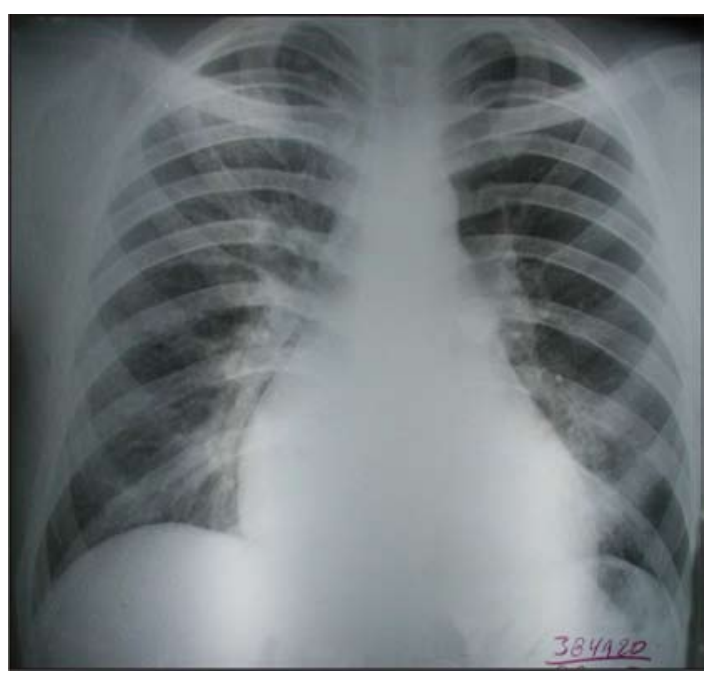

Figura 1. Radiografía posteroanterior del torax, mostrando ligera cardiomegalia a expensas de las cámaras derechas, ligera opacidad en región subclavicular izquierda y congestión pulmonar.
Al examen físico, se encontró paciente en aparente buen estado general, normotenso, con una frecuencia cardiaca de 44 y respiratoria de 18 al minuto, respectivamente; afebril, no se apreció edemas. En el área del precordio, se apreció choque de punta en el 5-6 espacio intercostal izquierdo (EII), bradicárdico, con ingurgitación yugular $(+)$ en 45 grados y un soplo sistólico mesocárdico grado III/VI. En el aparato respiratorio, se auscultó roncantes y sibilantes, en el tercio superior del hemitórax izquierdo.

Abdomen blando, no doloroso, hígado palpable a dos traveses de dedo por debajo del reborde costal derecho. Los exámenes auxiliares mostraron cifras hematológicas normales, así como las correspondientes a las funciones hepática y renal. La baciloscopía directa para bacilo de Koch fue negativa y la radiografía del tórax mostró cardiomegalia global y opacidad parenquimal, en la región subclavicular izquierda (Figura 1).

El electrocardiograma mostró bloqueo aurículo/ventricular de III grado, bloqueo completo de la rama derecha del haz de Hiss. Posteriormente, se le realizó un ecocardiograma, que fue informado como "Fracción de eyección 0,73 , fracción de acortamiento $34,3 \%$, dilatación de cámaras derechas, vena cava inferior dilatada, aneurisma del septum interventricular membranoso, con rotura y shunt de izquierda para derecha, insuficiencia tricuspídea funcional".

La fibrobroncoscopia mostró bronquio lobar superior derecho e izquierdo con mucosas congestivas. Examen de bacilo de Koch directo y Papanicolaou de aspirado broncoscópico fueron negativos.

Posteriormente, se realizó otro examen ecocardiográfico, informándose "Fracción de eyección ventricular: 0,70 , fracción de acortamiento $40 \%$, severa dilatación de cámaras derechas, rotura de seno de Valsalva no coronariano a aurícula derecha; vena cava inferior y venas suprahepáticas dilatadas con flujo sistólico reverso".

Con el diagnóstico de comunicación del ventrículo izquierdo al atrio derecho, el paciente fue programado para cierre quirúrgico de comunicación con circulación extracorpórea, por esternotomía 


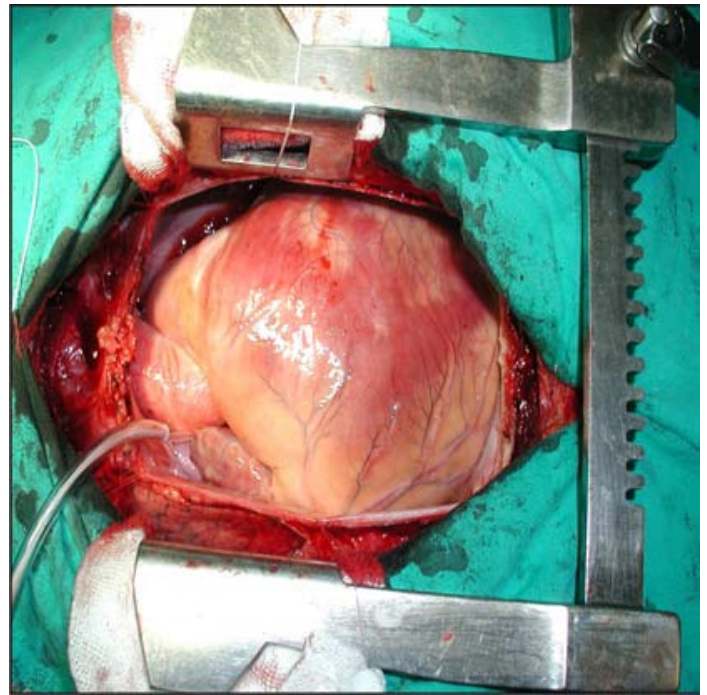

Figura 2. Esternotomía mediana: obsérvese la dilatación de las cámaras derechas.

media, encontrándose cardiomegalia a predominio de las cámaras derechas, derrame pericárdico de aspecto seroso de $100 \mathrm{~mL}$ de volumen, y una comunicación en el séptum membranoso en su porción aurículo-ventricular, a $10 \mathrm{~mm}$ por encima de la implantación de la valva septal de la válvula tricúspide. El defecto fue cerrado mediante la colocación de un parche de dacrón (Figuras 2 a 6).

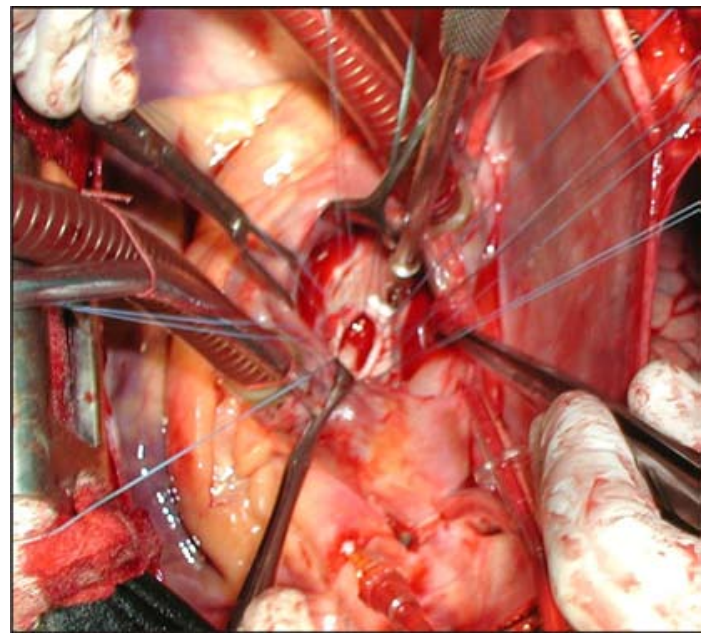

Figura 4. Obsérvese los bordes de la comunicación reparados con puntos de polileno.

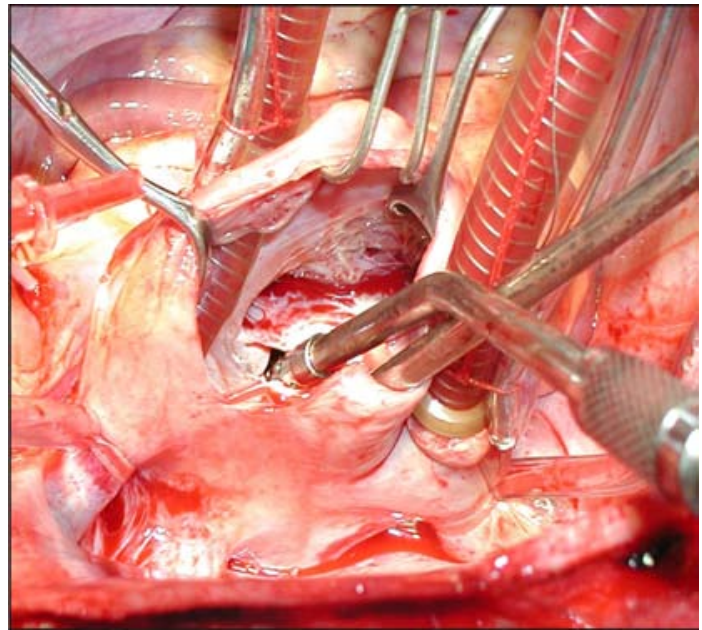

Figura 3. Abierta la aurícula derecha se observa la comunicación ligeramente por encima de la valva septal de la tricúspide, por donde ingresa el aspirador hasta la cámara ventricular izquierda.

\section{Evolución postoperatoria}

El ecocardiograma de control postoperatorio inmediato mostró que el defecto había sido cerrado completamente. No se auscultó soplos en el precordio, pero persistía el bloqueo aurículo-ventricular de III grado. El paciente presentaba tos con expectoración mucopurulenta y estertores roncantes

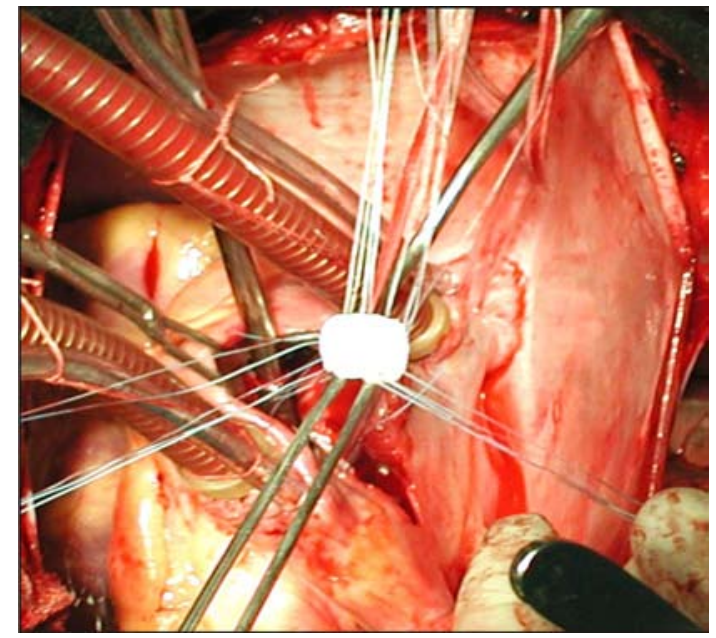

Figura 5. Se desliza un parche de dacrón mediante los hilos fijados al borde del defecto. 


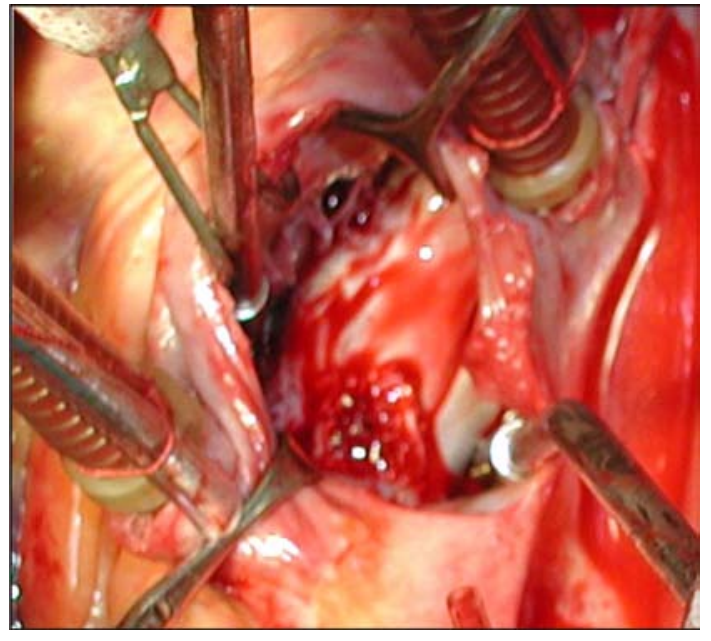

Figura 6. El parche ha sido colocado y la operación terminada.

y sibilantes, especialmente en tercio superior del hemitórax izquierdo, que continuó hasta el cuarto día, en el que se decidió realizar broncoscopia, con un tubo de Chevalier-Jackson 8-40, obteniéndose regular cantidad de secreción mucosa de color rosado y luego visualizándose en el bronquio lobar superior la presencia de un cuerpo extraño (fragmento de ladrillo), que se extrajo.

A partir de este momento, el paciente evolucionó favorablemente y fue dado de alta en condiciones satisfactorias, a los 9 días del postoperatorio.

\section{DISCUSIÓN}

El defecto ha sido reconocido muchos años antes, pero fue Gerbode $\left({ }^{1}\right)$ quien presentó los primeros cinco casos con estudio clínico completo, incluyendo cateterismo cardíaco, en quienes se hizo la reparación quirúrgica y fueron presentados ante la asociación Quirúrgica Americana, de Nueva York, en abril de 1958. Consiste en una comunicación entre el ventrículo izquierdo y la aurícula derecha, a través del séptum interventricular, pero por encima de la implantación de la valva septal de la tricúspide

Las variaciones anatómicas de este síndrome fueron resumidas por Perry y col. $\left({ }^{2}\right)$. Estos defectos pueden ser congénitos o adquiridos. Las formas congénitas comprenden menos del $1 \%$ de las enfermedades cardíacas congénitas. En general, tres variedades de comunicación deberían ser consideradas:

1. Fusión de la valva septal de la tricúspide a los bordes del defecto septal ventricular, asociada con una perforación de la valva. El shunt ocurre del ventrículo izquierdo, directamente dentro de la aurícula derecha.

2. Un defecto o hendidura de la valva de la tricúspide, en el punto de unión directamente sobre el defecto ventricular. El defecto ocurre del ventrículo izquierdo al ventrículo derecho y por regurgitación, dentro del atrio derecho.

3. Una combinación de estas dos lesiones, que permiten una variada proporción de sangre que entra al atrio derecho y el ventrículo derecho

La comunicación adquirida entre el ventrículo izquierdo y la aurícula derecha se origina en una perforación del séptum ventricular y puede ser como consecuencia de una endocarditis bacteriana $\left({ }^{3,4}\right)$, traumatismo $\left({ }^{5,6}\right)$, en el reemplazo de la válvula aórtica o mitral $\left({ }^{7-9}\right)$ y en el infarto del miocardio $\left({ }^{10}\right)$.

Ambas formas de comunicación -congénitas y adquiridas- están localizadas en el séptum membranoso interventricular, inferior a la cresta supraventricularis. Desde que el anillo tricuspídeo tiene una ubicación más apical que el anillo mitral, la valva septal de la válvula tricúspide queda 5 a 10 mm más apicalmente que la valva anterior de la válvula mitral. Esta disposición anatómica permite que la valva septal de la tricúspide divida el séptum ventricular membranoso en dos porciones:
A. Una porción supravalvular o atrioventricular.
B. Una porción infravalvular o interventricular $\left({ }^{11}\right)$.

Un defecto en la porción supravalvar resultará en una comunicación entre el ventrículo izquierdo y la aurícula derecha. Los defectos infravalvares resultarán en un defecto perimembranoso interventricular y establecen una comunicación entre ambos ventrículos. Los defectos de la valva septal o anterior de la tricúspide asociados a esta comu- 
nicación, tales como, perforación, fenestración, o hendidura de la valva, pueden permitir también la comunicación entre el ventrículo izquierdo y la aurícula derecha.

La transformación aneurismática de un defecto septal ventricular resulta de la adherencia del tejido valvar, sea de la valva septal o anterior de la tricúspide a los márgenes del defecto $\left({ }^{12}\right)$; las consecuencias hemodinámicas de la comunicación entre el ventrículo izquierdo y la aurícula derecha dependen de la magnitud del shunt, así como del tamaño del defecto $\left({ }^{12}\right)$. Teniendo en cuenta que la presión del ventrículo izquierdo es mucho mayor que la de la aurícula derecha, se presenta un pasaje de sangre hacia la aurícula derecha durante la sístole. Cuando el defecto es de gran tamaño, todo el flujo del shunt es regresado a ambos ventrículos, ocasionando sobrecarga biventricular y crecimiento de las cuatro cavidades. Cuando los defectos son muy pequeños, estos pueden quedar silentes por un período largo de tiempo $\left({ }^{13}\right)$.

Clínicamente, el paciente que es portador de una comunicación entre el ventrículo izquierdo y la aurícula derecha se presenta con síntomas de falla cardiaca congestiva y está documentado que la presencia de endocarditis bacteriana parece ser alta en comparación con los portadores de defecto septal ventricular $\left({ }^{2,12,14,15}\right)$. En el examen físico, es audible un soplo pansistólico, a menudo, asociado con un thrill palpable sobre el borde izquierdo del esternón. El diagnóstico no es posible sobre la base del examen físico solamente. La ecocardiografía bidimensional es hoy el examen de elección para establecer el diagnóstico. Los hallazgos deben ser distinguidos de los correspondientes a rotura de aneurisma del seno de Valsalva y defectos del cojín endocárdico y de la regurgitación tricuspídea. La ubicación supravalvular del defecto que comunica el ventrículo izquierdo con la aurícula derecha es más probable en defectos adquiridos que en los congénitos $\left({ }^{14}\right)$.

En el caso del paciente que presentamos, teniendo en cuenta su inicio súbito, sin antecedentes de endocarditis infecciosa, cirugía de cambio valvular ni manipulación de catéter intracardiaco -que son las causas más frecuentes de perforación adquirida de esta porción del séptum- y, por el contrario, coincidente con traumatismos que ocasionaron su primera atención por consulta de emergencia -después de haber sido víctima de traumatismo torácico cerrado, que ocasionó que fragmentos de cuerpo extraño fueran aspirados al bronquio lobar superior izquierdo-, solo podemos especular el probable origen traumático del defecto.

Concluyendo, podemos decir que el defecto de Gerbode es una cardiopatía de muy rara presentación y hay pocos casos comunicados en la literatura, siendo el nuestro el primer caso informado en la literatura nacional. Aunque, en la mayoría de los casos, el defecto es una consecuencia de la endocarditis infecciosa, los casos traumáticos suelen estar relacionados con traumatismo en el reemplazo de la válvula mitral o aórtica. En nuestro caso, cabe especular la posibilidad de que el traumatismo torácico cerrado haya ocasionado el defecto.

\section{REFERENCIAS BIBLIOGRÁFICAS}

1. Gerbode F, Hultgren H, Melrose D, Osborn J. Syndrome of left ventricular-right atrial shunt. Successful surgical repair of defect in five cases, with observation of bradycardia on closure. Ann Surg. 1958;148:433-6.

2. Perry EL, Burchell HB, Edwards JE. Congenital communication between the left ventricle and the right atrium; co-existing ventricular septal defect and double tricuspid orifice. Mayo Clin Proc. 1949;24:198-206.

3. Battin M, Fong LV, Monro JL. Gerbode Ventricularseptal defect following endocarditis. Eur J Cardiothorac Surg. 1991;5:613-4.

4. Velebit V, Schöneberger A, Ciaroni S, Bloch A, Maurice J, Christenson JT, et al. 'Acquired' left ventricular-to-right atrial shunt (Gerbode defect) after bacterial endocarditis. Texas Heart Inst J. 1995;22:100-2.

5. Kanber GJ, Fort ML, Treger A, Meadows WR, Sharp JT. Left ventricular-right atrial canal with aortic incompetence of probable traumatic origin. Am J Cardiol. 1967;20:879-83.

6. Venkatesh G, Lonn EM, Holder DA, Williams WG, Mulji A. Acquired left ventricular to right atrial communication and complete heart block following nonpenetrating cardiac trauma. Can J Cardiol. 1996;12:349-52.

7. KautznerJ, Munclinger MJ, Kozakova M. Acquired left ventricular-right atrial and right ventricular communication due to infective endocarditis after aortic valve replacement. Am Heart J. 1990;120:1233-4.

8. Seabra-Gomez R, Ross DN, Gonzales-Lavin L. Iatrogenic left ventricular-right atrial fistula following mitral valve replacement. Thorax. 1973;28:235-41. 
9. Silverman NA, Sethi GK, Scout SM. Acquired left ventricularright atrial fistula following aortic valve replacement. Ann Thorac Surg. 1980;30:482-6.

10. Jobic Y, Verdun F, Guillo P, Bezon E, Gilard M, Etienne $\mathrm{Y}$, et al. Postinfarction atrioventricular septal rupture. J Am Soc Echocardiogr. 1997;10:680-4.

11. Riemenschneider TA, Moss AJ. Left ventricular-right atrial communication. Am J Cardiol. 1967;19:710-8.

12. Wu MH, Chang CI, Wang JK, Lue HC. Characterization of aneurysmal transformation in perimembranous ventricular septal defects: an adhered anterior leaflet of tricuspid valve predisposes to the development of left ventricular-to-right atrial shunt. Int J Cardiol. 1994;47:117-25.

13. Selinger L, Werner K, Silber R, Nellessen U, Inselmann G. Natural history of a ventriculoatrial fistula after a gunshot injury in 1945. Ann Thorac Surg. 1998;65:1137-8.

14. Wasserman SM, Fann JI, Atwood JE, Burdon TA, Fadel BM. Acquired left ventricular-right atrial communication. Echocardiography. 2002;19(1):67-72.
15. Ochsenfahrt C, Friedl R, Hannekum A, Schumacher BA. Endocarditis after nipple piercing in a patient with a bicuspid aortic valve. Ann Thorac Surg. 2001;71(4):1365-6.

Manuscrito recibido el 22 de junio de 2007 y aceptado para publicación el 05 de setiembre de 2007.

\section{Correspondencia:}

Dr. José G. Somocurcio Vílchez

Departamento Académico de Cirugía

Facultad de Medicina - UNMSM

Av. Grau 755. Lima 1, Perú

Correo-e: jotagese@hotmail.com 